\title{
Los sistemas astrolátricos de Tenerife, La Gomera y Gran Canaria en los siglos XIV-XV
}

\author{
José Barrios García \\ Doctor en Historia. \\ Profesor titular de Análisis Matemático de la ULL. \\ http://doi.org/10.31939/bierehite/2019.07
}

\section{Nota preliminar}

El texto que sigue es, en esencia, una transcripción parcialmente editada de la conferencia que impartí en el Museo de Historia y Antropología de Tenerife, el día 5 de diciembre de 2019, en el marco de los talleres BIEREHITE¹ . Con anterioridad, ya había impartido esta conferencia en las II Jornadas de Estudios Bereberes (La Laguna, 16 de noviembre de 2017) y en el Ateneo Miraflores 3 (Santa Cruz de Tenerife, 1 de marzo de 2018).

Después de esta última fecha, la investigación sobre los procesos de sincretismo religioso relacionados con la Virgen de Candelaria en Tenerife, se ha visto enriquecida por un interesante trabajo de Lorenzo Santana ${ }^{2}$, donde documenta que la fecha de la celebra-

\footnotetext{
1 Quiero dar las gracias a los técnicos del MHAT y, especialmente, a José Antonio Torres, por la transcripción de la conferencia y su interés en publicarla. Yo mismo edité esa primera transcripción intentando mantener un cierto equilibrio entre la espontaneidad propia de una conferencia y la necesidad de aclarar algunas partes, estilizar el texto y evitar redundancias que en directo pasan desapercibidas.

2 Ver Santana (2018).
} 
ción de la Candelaria en agosto, en Tenerife, no permaneció fija, sino que estuvo fluctuando entre el 15 de agosto y el 8 de septiembre, al menos, durante el siglo XVII. Santana utiliza algunos de los resultados de esta conferencia para proporcionar una explicación que me parece muy acertada: la fecha fluctuaba por las tensiones soterradas entre mantener la fecha tradicional del 15 de agosto - promovida por los dominicos-y mantener una fecha cercana a las circunstancias reales del orto helíaco de Canopo - promovida por los naturales de la isla-. No obstante, en esta conferencia me mantuve fiel al contenido original de la misma y no incluí sus observaciones, que pueden verse en el trabajo citado ${ }^{3}$.

La Laguna, 15 de agosto de 2020.

\section{Conferencia}

¡Buenos días! Para justificar la presencia de un matemático en este ciclo de conferencias, podría decirles que cursé los estudios de doctorado en el Instituto de Ciencias Políticas y Sociales de la Universidad de La Laguna y, en el año 1997 presenté mi tesis doctoral sobre Los sistemas de numeración y calendarios de Tenerife y Gran Canaria en los siglos XIV y XV, codirigida por nuestro recordado Fernando Estévez ${ }^{4}$. Esta tesis fue el resultado de 12 años de investigación en los que tuve que buscar por los recovecos de la bibliografía, las noticias raras y dispersas que proporcionaban alguna información sobre mi objeto de estudio: en la práctica, un tema oculto en las fuentes y del que se sabía muy poco. La conferencia que les presento aquí es, básicamente, una parte de cómo ha evolucionado esa tesis hasta ahora 5 .

3 Ver, además, García-Bolaños-Paz (2019: 18-29).

4 Publicada en Barrios (2004).

5 Para una síntesis actualizada de los conocimientos matemáticos y astronómicos de los antiguos isleños en su contexto norteafricano, ver Barrios (2015). 
La información que encontré en mis primeros estudios sobre los conocimientos matemáticos y astronómicos de los antiguos habitantes de las islas estaba muy relacionada con su religión, lo que me empujó a estudiar los procesos de sincretismo religioso tras la conquista. Lo que voy a contarles aquí es lo que sabemos actualmente sobre los sistemas astrolátricos de guanches, gomeros y canarios, a través de los procesos sincréticos que se desarrollaron en estas tres islas después de la conquista. Unos procesos que nos llevan directamente a la Virgen de Candelaria en Tenerife, a la Virgen de la Candelaria en Chipude y a la Virgen del Pino en Teror. Mis estudios me han llevado a concluir que estos tres cultos católicos son sendas cristianizaciones del mismo sistema astrolátrico norteafricano y ese será el contenido de mi charla.

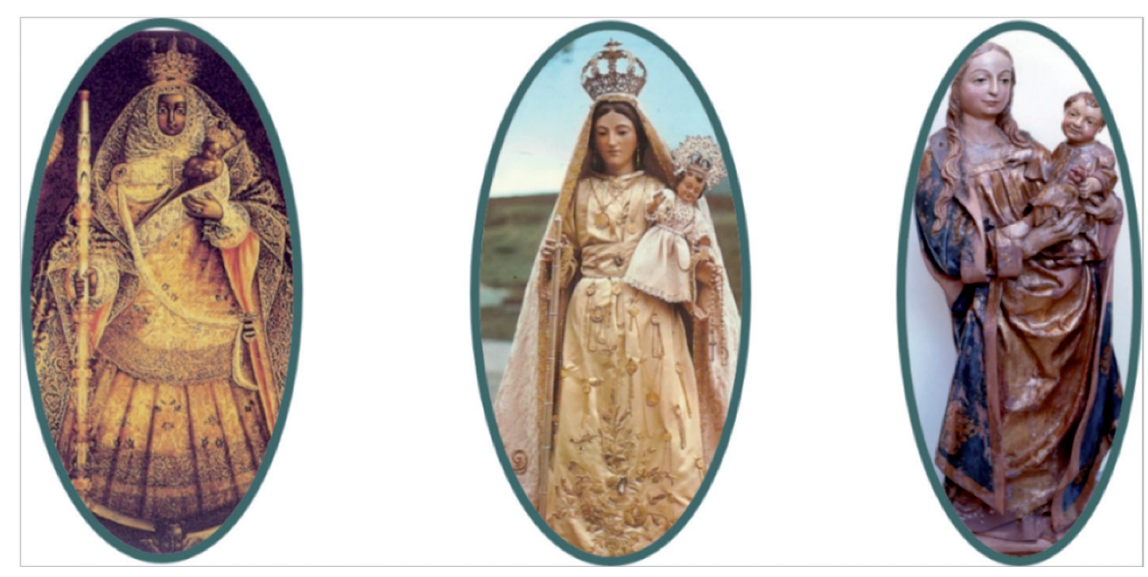

Fig. 1. Virgen de Candelaria, Tenerife (i). Virgen de la Candelaria,

Chipude (c). Virgen del Pino, Teror (d).

Lo que acabo de decir tiene implicaciones muy importantes. Ustedes se preguntarán: ¿en qué se basa para decir eso? Bueno, pues a lo largo de esta conferencia les voy a proporcionar un conjunto abundante y variado de pruebas arqueológicas, astronómicas, antropológicas, documentales, etnográficas y paralelos norteafricanos, que, en conjunto, forman un entramado que demuestra lo que estoy diciendo, más allá de toda duda razonable. 
Por comodidad, voy a dividir la charla en tres partes. En la primera repasaré algunas cuestiones astronómicas básicas necesarias para entender lo que sigue. En la segunda expondré lo que sabemos sobre los sistemas astrolátricos norteafricanos. En la tercera veremos que lo que sucede en las islas es totalmente congruente con la información norteafricana, lo que genera un sistema interpretativo completo y cerrado que deja poco margen para la duda.

\section{Primera parte. Preliminares astronómicos ${ }^{6}$}

Para empezar, entenderemos por astrolatría, el culto a los astros entendidos como divinidades y por sistemas astrolátricos, los sistemas teóricos y prácticos relacionados con los cuerpos astrales entendidos como divinidades. Esto incluye mitos de origen, prácticas religiosas, observaciones astronómicas, sistemas calendáricos... todo este tipo de cosas. Estas cuestiones son muy importantes en todo el Norte de África y aquí también, pero se les ha prestado muy poca atención. De hecho, estos sistemas astrolátricos pueden influir y explicar la organización económica, política, social y territorial de los diversos grupos humanos. Veremos por qué digo algo tan sorprendente.

Todas las sociedades agrícolas y ganaderas -y las canarias lo eran- han necesitado conocer con exactitud el paso de las estaciones para saber cuándo plantar, cuándo aparear el ganado, etc. No podemos sembrar en mayo porque el calor del verano secará las plantas: debemos sembrar en otoño, cuando comienzan las lluvias. De la misma manera, debemos aparear el ganado por San Juan -finales de junio/julio-, para que los baifos nazcan con el verde del invierno y ellos y sus madres tengan comida suficiente para sobrevivir. Cuestiones básicas que vienen del Neolítico.

6 Una descripción más detallada y precisa de los ciclos calendáricos y las técnicas astronómicas que menciono en este apartado puede verse en los capítulos 3, 4 y 5 de Barrios (2004). 


\begin{tabular}{|c|c|c|c|}
\hline Año & Duración & Determinación & \\
\hline $\begin{array}{l}\text { Solar } \\
\text { Sideral }\end{array}$ & $\begin{array}{l}365 \text { días } \\
365 \text { días }\end{array}$ & $\begin{array}{l}\text { Mov. en el horizonte } \\
\text { Fases de las estrellas }\end{array}$ & En fase con las estaciones \\
\hline $\begin{array}{l}\text { Lunar sinódico } \\
\text { Lunar sideral }\end{array}$ & $\begin{array}{l}354 \text { días } \\
328 \text { días }\end{array}$ & $\begin{array}{l}12 \text { meses sinódicos } \\
12 \text { meses siderales }\end{array}$ & Desfase con las estaciones \\
\hline $\begin{array}{l}\text { Lunisolar } \\
\text { Lunisideral }\end{array}$ & $\begin{array}{l}\text { 12/13 m. sinódicos } \\
12 / 13 \mathrm{~m} \text {. sinódicos }\end{array}$ & $\begin{array}{l}\text { Ajustado por el sol } \\
\text { Ajustado por las estrellas }\end{array}$ & En fase con las estaciones \\
\hline
\end{tabular}

Fig. 2. Tipos básicos de calendario.

Si queremos llevar el control de las estaciones, podemos hacerlo de varias maneras. La más sencilla es controlar el movimiento del sol en el horizonte, lo que da lugar a un año solar de 365 días, aproximadamente. Ahora mismo (comienzos de diciembre), el punto del horizonte por donde sale el sol se está moviendo hacia el sur de forma cada vez más lenta, más lenta, más lenta... y se parará el 21 de diciembre (solsticio de invierno), cuando alcance su extremo sur. A partir de este momento, el orto del sol empezará a moverse de nuevo hacia el norte, primero muy despacio y después cada vez más rápido, de forma que saldrá por el este el 21 de marzo lequinoccio de primaveral y alcanzará su extremo norte el 21 de junio (solsticio de verano). En la latitud de Canarias, el ángulo que recorre el orto del sol de un solsticio a otro es de unos de $54^{\circ}$ - que es mucho- y hemos podido documentar arqueológicamente diversos marcadores solsticiales, algunos muy sofisticados, que prueban el uso de este tipo de técnicas calendáricas y astronómicas en las islas ${ }^{7}$.

En realidad, el control de las estaciones lo podemos hacer por el sol o por las estrellas. Como la Tierra se mueve alrededor del sol,

7 Por ejemplo, los marcadores solsticiales de Cuatro Puertas en Gran Canaria (Barrios 1993b) o las Toscas del Guirre en La Gomera (Barrios-Hernández-Trujillo 2014). Sorprendentemente, en Tenerife no se han encontrado todavía marcadores claros. 
las estrellas que vemos por la noche van cambiando, apareciendo y desapareciendo a lo largo del año. Por eso podemos fijarnos en una estrella, por ejemplo, Sirio - como hacían los egipcios-y observar su periodo de aparición, desaparición y reaparición en el cielo, lo que da lugar a un año sideral de 365 días, aproximadamente, con un pequeño desajuste de unos 20 minutos con respecto al año solar.

Como acabamos de ver, para periodos largos - anuales- podemos utilizar tanto el sol como las estrellas, sin embargo, para periodos cortos prácticamente todas las sociedades utilizan la luna y los meses lunares, teniendo en cuenta que los meses lunares podemos medirlos de dos maneras, según sean meses sinódicos o meses siderales. Los meses sinódicos se cuentan por el paso de la luna por el sol - de luna nueva a luna nueva o de luna llena a luna llena-y duran 29.5 días aproximadamente, de forma que 12 meses lunares sinódicos hacen un año lunar sinódico de 354 días, con un desfase de 11 días respecto del año solar. Los meses siderales se cuentan por el paso de la luna por una misma estrella y duran 27.3 días aproximadamente. Por tanto, si utilizamos un año formado por 12 meses lunares siderales, obtenemos un año lunar sideral de 328 días, aproximadamente.

¿Cómo podemos compaginar los años lunares con los años solares o siderales y el paso de las estaciones? El mes lunar más utilizado es el sinódico, contado de luna nueva a luna nueva lo de primer creciente a primer creciente). Si contamos el tiempo por meses lunares sinódicos, supongamos que este año el día del solsticio de verano coincide con luna nueva y empezamos a contar el tiempo a partir de aquí. Un año lunar más tarde (doce lunas más tarde),

8 En el caso de que estemos utilizando años siderales, pensemos que el orto helíaco de la estrella coincide con luna nueva. El resto del razonamiento es análogo, cambiando solsticio de verano por orto helíaco. 
han pasado 354 días y nos faltan 11 días para el solsticio de verano, pero decimos: "bueno, no pasa nada, prácticamente estoy en la misma fecha". A continuación, dejamos transcurrir otro año lunar y al finalizar nos habremos adelantado otros 11 días, por lo que ahora faltan 22 días para el solsticio de verano. Si dejamos pasar otro año lunar, finalizará 33 días antes del solsticio de verano y así sucesivamente. ¿Qué podemos hacer para evitar el desfase del año lunar con el año solar o sideral y las estaciones? La respuesta en muchas ocasiones ha sido introducir un mes lunar intercalar para compensar el adelanto, lo que da lugar a los llamados años lunisolares (en su caso, lunisiderales).

Existen varios sistemas para introducir meses intercalares. Uno de los más antiguos es la octaetéride, que intercala 3 lunas sinódicas en 8 años solares o siderales, pero el más conocido es el ciclo metónico, que intercala 7 lunas sinódicas en 19 años solares o siderales y cuyo uso afecta a aspectos importantes de nuestra vida porque es el que utiliza la Iglesia Católica para ajustar las fechas móviles del calendario, como la Cuaresma o la Semana Santa?.

De forma similar, los calendarios basados en años lunares siderales pueden ajustarse al año solar o sideral mediante distintas formas de intercalación. En mi tesis pude documentar algunos de los calendarios lunisolares o lunisiderales utilizados en las islas en los siglos inmediatamente anteriores a la conquista, algunos documentados con mucha claridad y otros mucho más ocultos en las fuentes ${ }^{10}$.

\footnotetext{
9 El Domingo de Resurrección - la fiesta más importante de la liturgia católica-se fija como el primer domingo posterior a la primera luna llena igual o posterior al equinoccio de primavera. El resto de fiestas móviles de la Iglesia se determina en relación a este domingo.

10 Barrios (2004: caps. 9 y 15).
} 
Pero aquí me quiero fijar en el calendario sideral, es decir, el que lleva el control del año por la desaparición y la reaparición de las estrellas. Como dijimos, las estrellas que vemos en el cielo van cambiando poco a poco cada noche. Por ejemplo, esta noche al atardecer, si las nubes lo permiten, cuando el sol se ponga en el oeste podrán ver alguna estrella saliendo por el este. Es lo que llamamos el orto acrónico de la estrella: la estrella sale a la puesta del sol. Mañana, al ponerse el sol, a esa misma hora esa estrella estará un poquito más alta, pasado mañana un poquito más alta y así hasta que la veremos en el cenit a la puesta de sol. Con el paso de los días, esa estrella se irá acercando al sol, hasta que la veremos por última vez justo después de la puesta del sol. Es lo que llamamos la puesta helíaca de la estrella: la última vez que se ve la estrella antes de su periodo de desaparición. En los días siguientes la estrella no puede verse porque la oculta la luz del sol, así que desaparece del cielo durante un periodo de tiempo que varía según diversos factores, como su posición en la esfera celeste o su propio brillo. Para volverla a ver tendremos que trasladarnos al amanecer.

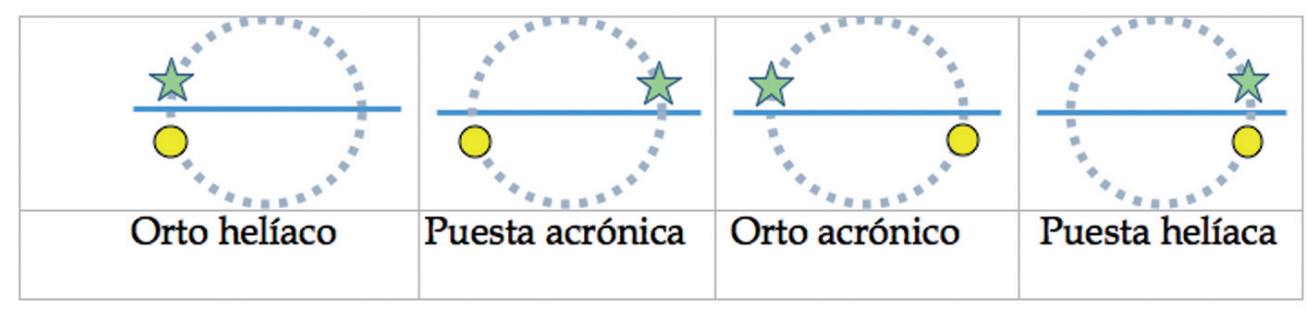

Fig. 3. Fases de las estrellas.

En efecto, con el transcurso del tiempo la estrella vuelve a separarse del sol - adelantándose a él-y la veremos aparecer durante un breve instante en el horizonte oriental, justo antes de la salida del sol. Es lo que llamamos el orto helíaco de la estrella: su primera aparición tras el periodo de invisibilidad. Con el paso de los días, la estrella se verá cada vez más alta al amanecer y se irá separando cada vez más y más del sol, hasta que la veremos ponerse a la salida del sol, momento que recibe el nombre de puesta acrónica: la estrella se pone al salir el sol. Estas cuatro posiciones relativas 
de las estrellas y el sol se denominan las fases de las estrellas (Fig. 3) y han sido utilizadas desde la Antigüedad como marcadores astronómicos y calendáricos ${ }^{11}$. Por ejemplo, Hesíodo utiliza las fases de las estrellas en su Trabajos y días para determinar distintos momentos del año apropiados para las actividades agrícolas, ganaderas, etc.

¿En qué fechas ocurren las fases de las estrellas? Dependen de cada estrella y, además, cambian a lo largo de los años debido a los movimientos seculares del cielo (precisión de los equinoccios, cambios en la oblicuidad de la eclíptica y los movimientos propios). De forma que las fases de cada estrella tienen lugar en una fecha propia que varía a lo largo del tiempo, por lo que se necesita calcularlas para cada estrella, para cada época, para cada fase y para cada latitud del observador ${ }^{12}$.

El modelo clásico para calcular las fases - se los comento muy rápidamente- hacía una estimación del orto o la puesta helíaca de la estrella utilizando un ángulo mínimo de separación del sol, llamado arcus visionis, calculado de forma empírica. Es un método muy antiguo y muy poco preciso, afortunadamente, en 1985, un astrónomo norteamericano que trabajaba para la NASA, Bradley Shaffer, publicó un pequeño programa de ordenador para hacer este cálculo de una forma más ajustada a los modelos astronómicos modernos. El programa, mucho más exacto, utiliza las coordenadas de los astros, la posición del observador, la calidad del cielo, las condiciones atmosféricas, etc. De esta forma diseñó un modelo de cálculo que contrastó con la observación in situ de más de 500 ortos y puestas helíacas ${ }^{13}$.

11 Simplifico la descripción de las fases. El orden en que se suceden puede variar de una estrella a otra dependiendo de varios factores.

12 Sobre la reconstrucción del cielo histórico de las islas, ver Barrios (2004: cap. 5).

13 Schaefer $(1985,1987)$. 


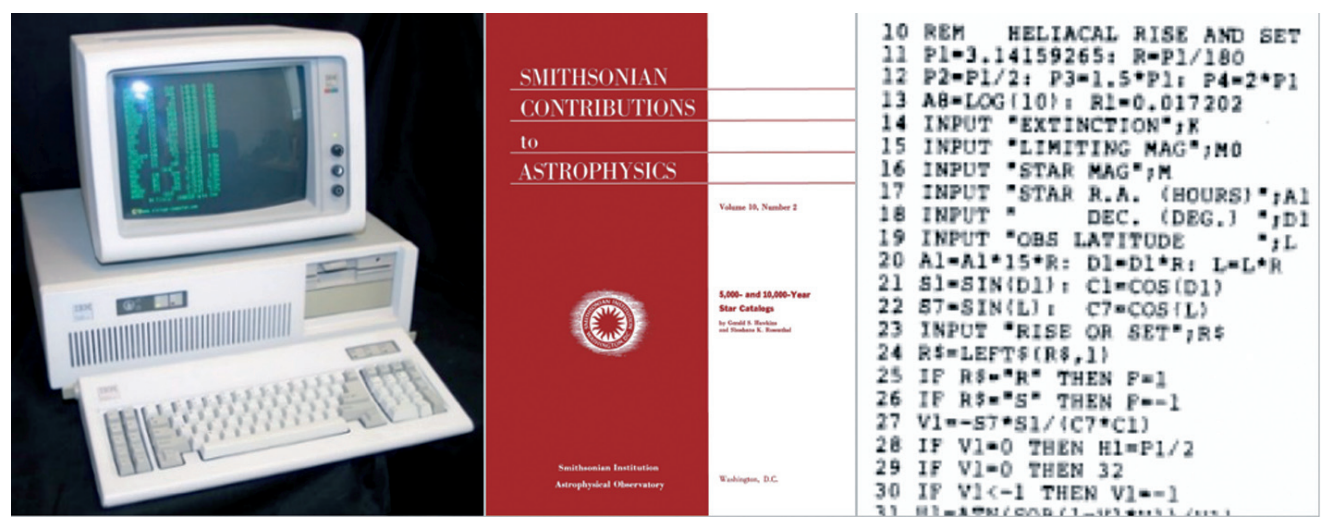

Fig. 4. IBM PC 8086 (i). Hawkins \& Rosenthal (1967) (c).

Comienzo del código en Schaefer (1985) (d).

¿Qué ocurría? Que el programa tenía una capacidad de cálculo limitada: utilizaba el lenguaje Basic de la época y había que hacer cada cálculo para cada estrella con una introducción individual de los datos. Se trataba, en fin, de un trabajo muy laborioso. ¿Qué hice en la tesis? pues sistematicé el modelo del profesor Schaefer utilizando los primeros ordenadores IBM que llegaron al mercado local a un precio relativamente asequible. En la imagen pueden ver un ordenador como el que yo tenía en esa época, con un disco duro de 20 MB (Fig. 4). Lo que hice fue adaptar el programa de Schaffer para usarlo en una hoja de cálculo y poder calcular al mismo tiempo los ortos y las puestas helíacas cada 500 años de un conjunto seleccionado de 24 estrellas, desde el año 1000 AC hasta el año 2000 DC (Fig. 5). Las coordenadas de cada estrella en cada época las tomé del catálogo de Hawkins \& Rosenthal (1967) que recoge las posiciones de las estrellas en los últimos 5000 años. Así produje una tabla de ortos y puestas helíacas de las 24 estrellas seleccionadas, en la latitud de Canarias, utilizando el calendario legal en vigor en cada momento ljuliano antes de la reforma gregoriana de 1582 y gregoriano después de $1582^{14}$.

14 Recientemente rehíce el cálculo de la tabla utilizando las coordenadas y los movimientos propios de las estrellas 2000 que proporciona la base de datos Simbad, alojada en el Centre de Données astronomiques de Strasbourg y obtuve resultados muy similares a los obtenidos en 1994. 


\begin{tabular}{|c|c|c|c|c|c|c|c|c|c|c|c|c|c|}
\hline ASTRO & Mag & $\begin{array}{c}1 \\
\text { Orto }\end{array}$ & $\begin{array}{c}\text { DC } \\
\text { Puesta }\end{array}$ & $\begin{array}{l}500 \\
\text { Orto }\end{array}$ & $\begin{array}{c}\text { DC } \\
\text { Puesta }\end{array}$ & $\begin{array}{l}1000 \\
\text { Orto }\end{array}$ & $\begin{array}{c}\text { DC } \\
\text { Puesta }\end{array}$ & $\begin{array}{l}1500 \\
\text { Orto }\end{array}$ & $\begin{array}{c}\text { DC } \\
\text { Puesta }\end{array}$ & $\begin{array}{l}1600 \\
\text { Orto }\end{array}$ & $\begin{array}{c}\text { DC } \\
\text { Puesta }\end{array}$ & $\begin{array}{l}2000 \\
\text { Orto }\end{array}$ & $\begin{array}{c}\text { DC } \\
\text { Puesta }\end{array}$ \\
\hline Achernar & 0.60 & \#\#\#\#\# & \#\#\#\# & \#\#\#\# & \#\#\#\#\# & \#\#\#\# & \#\#\#\#\# & $17 \mathrm{jul}$ & 8 ene & 25 jul & 20 ene & $19 \mathrm{jul}$ & $1 \mathrm{feb}$ \\
\hline Acrux & 1.10 & 5 nov & 15 jun & 1 nov & 8 jun & 21 nov & 1 jun & $7 \mathrm{dic}$ & 18 may & 1 ene & 18 may & \#\#\#\# & \#\#\#\# \\
\hline Alción & 2.96 & 21 may & & may & $8 \mathrm{abr}$ & 26 may & $11 \mathrm{abr}$ & 8 may & $15 \mathrm{abr}$ & 8 jun & br & 13 jun & $30 \mathrm{abr}$ \\
\hline Aldebarán & 1.06 & 30 may & $20 \mathrm{abr}$ & 1 jun & $23 \mathrm{abr}$ & 3 jun & $26 \mathrm{abr}$ & 5 jun & $28 \mathrm{abr}$ & 15 jun & 9 may & 20 jun & 14 may \\
\hline Alnilam & 1.75 & 7 jun & $26 \mathrm{abr}$ & 8 jun & $29 \mathrm{abr}$ & 30 jun & 1 may & 1 jul & 2 may & 1 jul & 13 may & $15 \mathrm{jul}$ & 17 may \\
\hline Altair & 0.89 & $24 \mathrm{dic}$ & $27 \mathrm{dic}$ & 6 dic & $29 \mathrm{dic}$ & $28 \mathrm{dic}$ & $31 \mathrm{dic}$ & $29 \mathrm{dic}$ & 1 ene & ene & 12 ene & 13 ene & 16 ene \\
\hline Antares & 1.22 & $21 \mathrm{nov}$ & 11 oct & 24 nov & 15 oct & $\mathrm{v}$ & & $2 \mathrm{dic}$ & 23 oct & dic & 2 nov & $18 \mathrm{dic}$ & 9 nov \\
\hline Arturo & 0.24 & 30 sep & 17 oct & 3 oct & 19 oct & & oct & 9 oct & loct & 20 oct & nov & & 5 nov \\
\hline Betelgeuse & 0.10 & 21 jun & 9 may & 3 jun & 10 may & 25 jun & 13 may & 26 jun & 15 may & $7 \mathrm{jul}$ & 25 may & $11 \mathrm{jul}$ & 30 may \\
\hline Canopo & -0.86 & 23 ago & $17 \mathrm{abr}$ & ago & $16 \mathrm{abr}$ & go & $13 \mathrm{abr}$ & ago & $12 \mathrm{abr}$ & 8 ago & $2 \mathrm{abr}$ & 30 ago & $23 \mathrm{abr}$ \\
\hline Capella & 0.21 & 5 may & 12 may & 10 may & 18 may & 14 may & 22 may & 19 may & 28 may & 30 may & 7 jun & 7 jun & 4 jun \\
\hline Castor & 1.99 & $1 \mathrm{jul}$ & & 4 jul & fiun & 7 jul & & $10 \mathrm{jul}$ & & & 23 jun & ul & 29 jun \\
\hline Deneb & 1.3 & $24 \mathrm{dic}$ & 31 ene & $23 \mathrm{dic}$ & 31 ene & dic & 2 feb & 1 dic & b & ic & 3 feb & e & $17 \mathrm{feb}$ \\
\hline Fomalha & 1.29 & $19 \mathrm{mar}$ & 4 ene & $24 \mathrm{mar}$ & 9 ene & $7 \mathrm{mar}$ & 13 ene & $31 \mathrm{mar}$ & 18 ene & $10 \mathrm{abr}$ & 29 ene & $15 \mathrm{abr}$ & 4 feb \\
\hline Hadar & 0.86 & 15 nov & 9 jul & 22 nov & $6 \mathrm{jul}$ & 2 dic & 30 jun & $13 \mathrm{dic}$ & 24 jun & $26 \mathrm{dic}$ & 2 jul & 14 ene & 25 jun \\
\hline Mimos & 1.50 & 4 nov & 24 jun & noy & jun & 6 nov & jun & . & & & 6 jun & & 10 jun \\
\hline Pollux & & & & & & $9 \mathrm{i}$ & 13 & 1. & 16 jun & 1 & un & 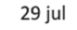 & 3 jul \\
\hline Proción & 0. & $10 \mathrm{jul}$ & un & 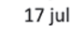 & & 19 & 5 jun & إ & jun & & 17 jun & 5 ago & 21 jun \\
\hline Régulo & 1.34 & 12 ago & 9 jul & 15 ago & 12 jul & 18 ago & $14 \mathrm{jul}$ & 1 ago & $17 \mathrm{jul}$ & 31 ago & $27 \mathrm{jul}$ & 6 sep & 2 ago \\
\hline Rigel & 0.34 & 23 jun & $23 \mathrm{abr}$ & 24 jun & $25 \mathrm{abr}$ & 25 jun & $27 \mathrm{abr}$ & 26 jun & $28 \mathrm{abr}$ & $6 \mathrm{jul}$ & 8 may & $10 \mathrm{jul}$ & 13 may \\
\hline Rigil K & & 24 nov & & & & 12 & 9 jul & $3 \mathrm{dir}$ & 1 jul & ne & $10 \mathrm{jul}$ & 25 ene & 2 jul \\
\hline Sirio & & & $16 n$ & 14 & & & may & 17 iul & 19 may & & & & 1 jun \\
\hline Spica & 1.2 & 5 oct & 28 ago & 8 oct & 31 & 12 oct & 3 sep & 15 oct & $6 \mathrm{sep}$ & 25 oct & $16 \mathrm{sep}$ & 31 oct & $23 \mathrm{sep}$ \\
\hline ega & 0.14 & 25 nov & 6 ene & $25 \mathrm{nov}$ & 7 ene & $25 \mathrm{nov}$ & 6 ene & $25 \mathrm{nov}$ & 6 ene & $4 \mathrm{dic}$ & 16 ene & $8 \mathrm{dic}$ & 20 ene \\
\hline
\end{tabular}

Fig. 5. Tabla de ortos y puestas helíacas en la latitud de Canarias

(calendario legal juliano/gregoriano).

Para ilustrar la situación, en la latitud de Canarias, en los años 1000-1500 DC, el orto helíaco de Sirio, la estrella más brillante del cielo, estaba ocurriendo a mediados de julio y su puesta helíaca a mediados de mayo, aproximadamente. Por su parte, el orto de Canopo, la segunda estrella más brillante del cielo, estaba ocurriendo a mediados de agosto y su desaparición tenía lugar a mediados de abril. El orto de las Pléyades, representadas por Alción, tenía lugar a finales de mayo y su puesta a mediados de abril.

\section{Segunda parte. Los sistemas astrolátricos norteafricanos}

Iniciamos ahora la segunda parte de la conferencia. ¿Qué sabemos sobre los sistemas astrolátricos en el norte de África? Las fuentes antiguas son unánimes al señalar que los antiguos bereberes eran astrólatras. Por ejemplo, Heródoto, en el libro IV de su Historia, dice que [los libios] solo inmolan al sol y a la luna. Todos los libios hacen sacrificios a estas dos divinidades. Cicerón, Diodoro Sículo, Plinio el Viejo, Macrobio o, más modernamente, Ibn Jaldún o Luis 
Mármol, todos ellos señalan la importancia de la astrolatría entre las poblaciones norteafricanas (Fig. 6).

\begin{tabular}{|c|c|}
\hline $\mathrm{AC}$ & \\
\hline $\mathrm{C}^{\text {Siglo }}$ & Cicerón $D e \quad R e$ \\
\hline $\mathrm{C}^{\text {Siglo }}$ & $\begin{array}{cc}\text { Diodoro } & \text { Sícul } \\
\text { Bibliotheca Historica } & \text { III.57 }\end{array}$ \\
\hline $\mathrm{C}^{\text {Siglo }}$ & $\begin{array}{r}\text { Plin } \\
\text { storia II. }\end{array}$ \\
\hline$C$ & Catumli \\
\hline Siglo & $\begin{array}{l}\text { Ibn Jaldún Kitāb a } \\
\text { Tbār VI.89 }\end{array}$ \\
\hline $\mathrm{C}$ & $\begin{array}{c}\text { Luis } \mathrm{Má} \\
\text { Descripción general de }\end{array}$ \\
\hline
\end{tabular}

Fig. 6. Fuentes clásicas sobre la astrolatría norteafricana.

Los estudios modernos, también han hecho referencia a la astrolatría norteafricana. Por ejemplo, los trabajos clásicos de René Basset (1910), Gibert Charles-Picard (1954) o Gabriel Camps (1980, 1988) dedican un espacio más o menos importante a la religión astral. No obstante, la persona que mejor ha estudiado este tema entre las poblaciones norteafricanas modernas es la profesora Viviana Pâques (1920-2007), adscrita al Instituto de Etnología de París, que publicó en 1964 su tesis doctoral, titulada: L'arbre cosmique dans la pensée populaire et dans la vie quotidienne du nord-ouest africain. Un libro que compré hace muchos años en París y que contiene muchas claves sobre la antropología antigua de las islas (Fig. 7).

La profesora Viviana Pâques, llevó a cabo en los años 50-70 un trabajo de campo excelente en el norte de África, extensísimo: trabajó en el Fezzan, Sahara, Níger, Mali, Argelia y Túnez. La idea original 
de su tesis era estudiar el mundo espiritual de las poblaciones negras que habían sido esclavizadas y llevadas al norte de África, entendiéndolas como un subgrupo reducido y específico. Para su sorpresa, lo que encontró fue que este subgrupo poblacional no tenía un mundo espiritual particular: su mundo espiritual era extensivo al resto de las poblaciones norteafricanas. No había diferencias: la concepción del mundo era la misma para todas las gentes del norte y oeste de África. De hecho, advierte explícitamente que esta concepción es tan característica [para un antropólogo], como puede serlo un bifaz típico para un arqueólogo ${ }^{15}$. Es decir, después de empezar una investigación partiendo de otras premisas, encuentra un sistema cosmogónico común a todo el norte de África, ya sean poblaciones negras esclavizadas o poblaciones esclavizadoras. Todos tienen el mismo sistema y lo documenta en este libro mediante un impresionante trabajo de campo.

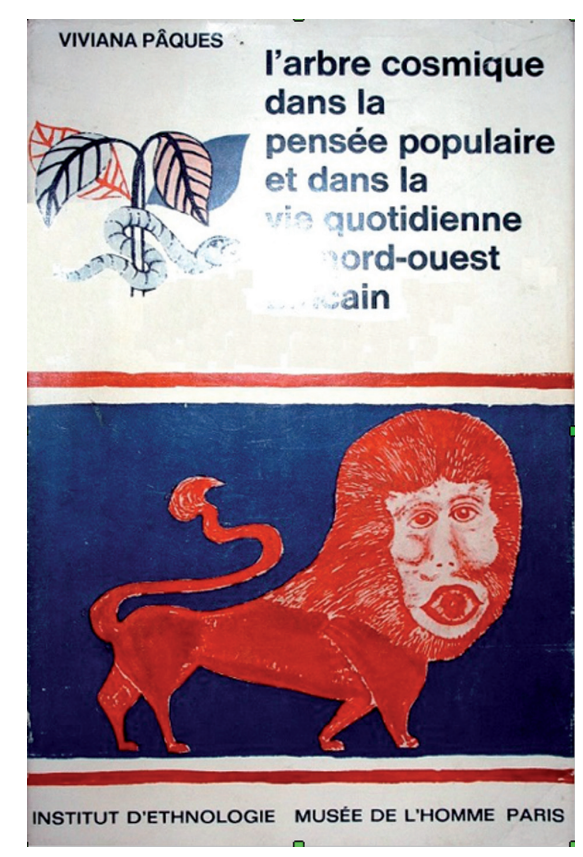

Fig. 7. Cubierta de la primera edición (Paris, 1964).

\footnotetext{
15 En dépit de toutes les différences extérieures ou locales, le monde spirituel africain est aussi défini, aussi reconnaissable que peut l'être en archéologie préhistorique un biface typique dont on suit l'expansion territoriale à travers les modifications dues à la matière première ou au génie individuel de l'artisan (Pâques 1964: 10).
} 
En este sistema, Dios creó el universo por la explosión de Canopo, la estrella primigenia, la más grande, la más antigua. Huevo primordial, madre de todas las estrellas, de su explosión surge el universo que conocemos. De la matriz de Canopo surgió la serpiente triple - que vemos en la portada del libro- y otras seis estrellas. El Universo involucionó cuando un héroe mató a la serpiente. Este héroe descendió a la Tierra por el triple árbol cósmico formado por el cuerpo de la serpiente decapitada. Más tarde, ese héroe, ascendió por el mismo árbol cósmico en ocasión de un segundo sacrificio de la serpiente. Todos esos sucesos míticos ocurrieron cuando el sol estaba en una determinada posición en relación con Canopo, para la explosión, y con las Pléyades, para el sacrificio ${ }^{16}$.

Realmente sorprendente. Cuando leí todo esto me quedé asombrado y lo cierto es que lo documenta de forma exhaustiva con todas las variantes propias de las diferentes zonas que cubrió en su trabajo de campo. Aquí solo les presento una pequeña síntesis de algunos aspectos importantes de su trabajo en relación con Canarias. No digo que esto sea exactamente lo que encontremos aquí, pero hay cosas que sí podemos ver en las islas. El papel fundamental de Canopo está detrás de las tres vírgenes que les mencioné y va a quedar muy claro en esta conferencia. El papel del triple árbol cósmico también lo podemos vislumbrar en el Pino de Teror, por ejemplo. Hay cosas, flashes que podemos ver.

Por otra parte, me resulta muy llamativo que no se trate de un sistema cosmogónico que vive al margen de la vida cotidiana: todo lo contrario. Lo que dice y documenta Viviana Pâques en su trabajo es que el sistema permea por toda la sociedad y define un conjunto de reglas que organiza todos los aspectos de la vida tradicional en el norte de África: la división del tiempo y los ritmos de los movimientos celestes, la división del año agrícola por medio de ciertas

16 Pâques (1964: 673). 
constelaciones, las estructuras políticas, territoriales y sociales -ni más ni menos-y, en general, el diseño de todo aquello que pueda reflejar este sistema en la vida diaria. Con respecto a su origen, opina que la coherencia interna, originalidad y distribución del mito sugieren que se originó en una fecha muy antigua en una comunidad agrícola, en territorio subdesértico, quizás en alguno de los oasis saharianos donde estas concepciones se conservan mejor ${ }^{17}$.

Todo esto es absolutamente sorprendente. Uno lo lee y se pregunta, ¿qué quiere decir todo esto? A continuación, veremos que los que nos cuenta Viviana Pâques sobre el pensamiento popular y la vida cotidiana de las poblaciones norteafricanas a mediados del siglo XX tiene mucho que ver con lo que sucedía en Canarias seis siglos antes.

\section{Tercera parte. Los sistemas astrolátricos canarios}

¿Y qué es lo que nos encontramos en Canarias? ¿Qué dicen las fuentes escritas? Cuando uno lee las fuentes más antiguas, no las fuentes mediatizadas por la conquista católica de finales del XVI-XVII: Espinosa, Abreu, Torriani, etc. Me refiero a las primeras de todas, cuando todavía las islas no estaban conquistadas. Por ejemplo, el papa Urbano $V$ señala en una bula muy conocida del año 1379 que le habían informado de que en Canarias y demás islas adyacentes, no teniendo más ley ni secta que la adoración del sol y la luna sería muy fácil reducirlas a la fe de Cristo. El historiador bereber Ibn Jaldún (1332-1406), hablando de cautivos canarios vendidos al sultán de Marruecos hacia el año 1370, dice que sus habitantes, los canarios, se alimentaban de cebada, su ganado se componía de cabras, su única práctica de devoción, consistía en postrarse ante el sol en el momento de su aparición. Alvise Cà da Mosto (1432-1483),

17 Pâques (1964: 676). 
un joven comerciante veneciano que pasó por las islas en 1455 , al hablar de Tenerife - antes de su conquista - dice que sus habitantes no tienen fe, sino que algunos adoran al sol, otros a la luna y algunos planetas. Diogo Gomes de Sintra, un marino portugués que pasó por las islas a mediados del siglo XV, al hablar de Tenerife y La Palma - también antes de su conquista- señala que los habitantes del lugar se llaman canarios y son numerosísimo pueblo, adoran al sol como un dios ${ }^{18}$.

Son noticias muy claras, muy directas, de la astronomía indígena que justifican de sobra el estudio de los sistemas astrolátricos canarios. Vamos a ver, entonces, cómo se reflejan o qué podemos saber de estos sistemas astrolátricos. Y la vía para conocer cómo funcionaban estos sistemas en las islas, lo que nos ha dejado más información sobre ellos, son los procesos de sincretismo religioso.

\section{Un problema calendárico: la Candelaria y las fiestas de agosto $^{19}$}

En Tenerife, la Candelaria y las fiestas de agosto plantean un problema calendárico muy importante, al que se le ha prestado muy poca atención. Cuando hablamos de las fiestas de la Candelaria todo el mundo asume que son fiestas guanches cristianizadas que tenían lugar a mediados de agosto. Pero, ¿por qué a mediados de agosto?

Si leemos las fuentes, varios historiadores señalan que los guanches celebraban algunas fiestas, que contaban el tiempo de la

18 Las referencias de las fuentes mencionadas pueden verse en Barrios (2004: 48-50).

19 Los detalles y referencias de este apartado pueden verse en Barrios (2004: cap. 15). 
luna con nombres diferentes y que el mes de agosto se llamaba beñesmer. El padre Espinosa dice que en la playa de Abona se veían luces y los guanches hacían procesiones y que esas fiestas, esas procesiones, tenían lugar principalmente por las fiestas de la Asunción de Nuestra Señora, es decir, a mediados de agosto. Marín de Cuba, el médico de Telde, nos da una información muy interesante y muy precisa. En su manuscrito de 1687 señala que los guanches:

... contaban el año por las sementeras y lo llamaban era y los tenían rallados y señalados en tablas y lo empezaban cerca de agosto, llamado beñesmer, en la primera luna y por 15 días continuos hasta la oposición hacían grandes fiestas.

Y en el manuscrito de 1694, dice:

Hacían sus fiestas como los canarios al fin de la era o año, empezado en la luna de agosto, llamado beñesmer.

Bueno, todos estos datos son muy precisos y todos coinciden en destacar la importancia que tenía la luna de agosto entre los guanches. La pregunta es: ¿cómo determinaban los guanches la luna de agosto? Si, por decirlo así, los años lunares van rotando por el calendario solar o sideral, ¿cómo sabían cuál era la luna correspondiente al mes de agosto castellano? Que yo sepa, el primero que se planteó ese problema fue Antonio Cubillo en un libro que publicó en 1985, Antropónimos guanches y bereberes, donde señala que saber cómo calculaban los guanches la luna de agosto es un problema técnico, astronómico ${ }^{20}$. En este sentido, recoge que en el Ahaggar argelino, el mes de agosto se llama igual que la estrella 
Canopo porque la estrella reaparece a finales de agosto tras su periodo de invisibilidad y concluye que los guanches podrían haber utilizado la reaparición de Canopo para determinar la fecha del 15 de agosto. Es decir, se plantea el problema y le da una posible solución, pero advierte que es necesario confirmar que las fechas de Canopo en Canarias coinciden con las argelinas.

Cuando leí esto ya había empezado mi estudio de los calendarios de las islas, así que pensé: ¿es así o no es así? Hay que comprobarlo. Y eso fue lo que hice en 1994 en una comunicación que presenté en la II Conferencia de la Sociedad Europea para la Astronomía en la Cultura (SEAC), celebrada en Bochum, Alemania ${ }^{21}$. En la conferencia presenté una confirmación astronómica y etnográfica de la solución propuesta por Antonio Cubillo. Básicamente, reconstruí el cielo histórico de las islas y lo confronté con las fuentes escritas y los paralelos norteafricanos lo que me permitió demostrar la asociación de la Virgen de Candelaria con la estrella Canopo. ¿Por qué digo esto? Veamos muy sintéticamente - voy muy al grano- las noticias que hay en las fuentes de cómo interpretaron los guanches la aparición de la estatua de la Virgen en la playa de Chimisay, en términos de su propia cultura. Como quiera que haya sido ese suceso, hay tres versiones principales:

- El fraile franciscano Martín Ignacio de Loyola (155\#1606), que pasó por las islas hacia 1580, estuvo en la fiesta de la Candelaria y dice que los guanches la llamaban la madre del sol.

- Fray Alonso de Espinosa (1542-159\#), al que todos conocemos, escribe en 1594 que los guanches la llamaron Achmayex Guayaxerax, la madre del sustentador del cielo y tierra.

21 Barrios (1996). 
- Y alguien que me llama mucho la atención es Antonio de Viana (1578-165\#), porque en su poema sobre la conquista de la isla publicado en 1604, al narrar la aparición de la Virgen de Candelaria, afirma que cuando los guanches encontraron la estatua se reunieron y discutieron sobre su origen. Algunos apuntaron que la habría arrojado el mar, a lo que otros objetaron que de ser así estaría estropeada, lo que no era el caso. Después de varios pareceres, la discusión se cerró cuando los varones en quien más parte de prudencia había, dijeron ser del cielo alguna estrella, en traje de mujer hermosa y bella. Así se acabó la discusión.

¿Una estrella? Vamos a investigarla. Si utilizamos como guía la tabla que les presenté antes sobre los ortos y puestas helíacas de las 24 estrellas principales (Fig. 5), ¿qué estrella puede servir para identificar la luna de agosto? Lo normal sería que dicha luna esté determinada por la fase de una estrella, presumiblemente la más importante: su orto helíaco o reaparición en el cielo después del periodo de invisibilidad.

Por ejemplo, si vemos la tabla, en el año 1500 solo hay dos candidatas: una es Canopo - orto helíaco el 18 de agosto-y la otra es Régulo - orto helíaco el 21 de agosto-. Canopo, la segunda estrella más brillante del cielo, se oculta el 12 de abril y sale el 18 de agosto. Régulo, la estrella más brillante de la constelación de Leo, se oculta el 17 de Julio y sale el 21 de agosto. Son las dos únicas que podrían tener relación con mediados de agosto. Vamos a ver que la evidencia se inclina totalmente a favor de Canopo.

Si se fijan, Canopo se oculta en el cielo a mediados de abril y sale a mediados de agosto, y cuando uno analiza en las fuentes escritas el calendario festivo de los guanches se encuentra con que los 
guanches celebraban tres fiestas principales: una a mediados de agosto - que es la que les acabo de comentar y que todos conocemos-, otra a principios de febrero y otra a finales de abril.

De hecho, la trama del Poema de Viana está centrada en las fiestas de finales de abril. ¿Qué cuenta Viana? Pues cuenta que los guanches estaban celebrando las fiestas de finales de abril en La Orotava y Alonso Fernández de Lugo aprovechó de esta circunstancia - que conocía perfectamente- para desembarcar sin oposición en las playas de Añazo. En este sentido, a veces me gusta bromear diciendo que las Fiestas de Mayo en Santa Cruz no son más que una pervivencia de las fiestas guanches de finales de abril, en el sentido de que Lugo aprovechó la fiesta guanche para desembarcar en Santa Cruz.

En Gran Canaria pasó lo mismo. Si uno mira la fecha del desembarco de Juan Rejón -24 de junio de 1478-, está claro que utilizó la misma táctica: aprovechar que la isla estaba celebrando las fiestas del solsticio de verano, bien documentadas, para desembarcar sin oposición. Los castellanos conocían los calendarios indígenas y no eran tontos, así que aprovechaban los momentos apropiados para desembarcar. La estrategia estaba clara: cogerlos en fiestas, cogerlos desprevenidos.

Volviendo a Tenerife, si uno estudia las tres fiestas que acabo de mencionar, ve que están relacionadas con las fases de Canopo: a mediados de agosto tenía lugar el orto helíaco, a finales de enero/ principios de febrero el orto acrónico y a finales de abril la puesta helíaca. Así que hay una correlación directa entre estas tres fases de Canopo y las tres fiestas principales de la isla (Fig. 8). 


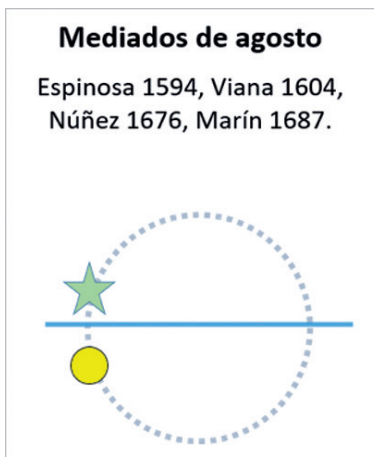

Orto helíaco

\section{Principios de febrero}

Acta de la cera 1497, Espinosa 1594, Viana 1604.

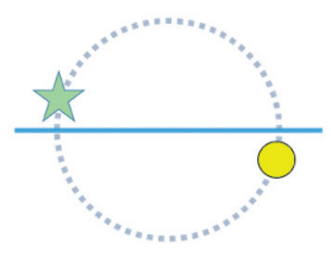

Orto acrónico

\section{Finales de abril}

Viana 1604, Núñez 1676.

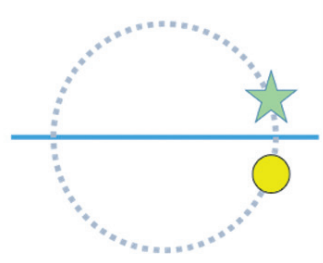

Puesta helíaca

Fig. 8. Calendario festivo de Tenerife correlacionado con las fases de Canopo.

En resumen: la fiesta de la Candelaria es la fiesta más importante de la isla, la celebración popular es el 15 de agosto y no el 2 de febrero - día de la fiesta católica-, hay una concentración de la población de la isla de origen guanche y está relacionada con la cueva de San Blas o cueva de Achbinico. Hace 25 años que sabemos que el culto católico a la Virgen de Candelaria es una adaptación del culto guanche a la estrella Canopo.

\section{La Gomera: Chipude y las fiestas de agosto}

Cualquiera que conozca un poco las fiestas de la Candelaria en Chipude y en Tenerife observará las grandes similitudes que existen entre ambas. En el año 2012, prospectando en Igualero con José Miguel Trujillo, del Museo Arqueológico de La Gomera, teníamos la Fortaleza de Chipude enfrente y le comenté el paralelismo entre las dos fiestas. Lo que me dijo definiría el rumbo de la investigación: En la Fortaleza de Chipude hay una cueva de San Blas. A lo que contesté: ¿Cómo? ¿Qué en la Fortaleza hay una cueva de San Blas? Al final, encontramos en Chipude un sistema exactamente igual al de Candelaria en Tenerife. 
Esta investigación la hice con Juan Carlos Hernández, arqueólogo, y José Miguel Trujillo, antropólogo, adscritos al Museo Arqueológico de La Gomera, cuyo interés y conocimiento de la isla fue clave para su desarrollo ${ }^{22}$. También resultó muy importante el trabajo de José Perera López sobre la toponimia gomera, donde recogió prácticamente cada topónimo de cada sitio y lo complementó con importantes y esclarecedoras tradiciones orales, todas muy interesante ${ }^{23}$.

Volviendo a nuestro tema... la fiesta de la Candelaria se celebra en Chipude con unas características formales muy semejantes a las de Candelaria en Tenerife: es una de las fiestas más importantes de la isla, la celebración popular también es el 15 de agosto y no el 2 de febrero, igualmente hay una concentración de la población de la isla y también tiene un origen muy cercano a la conquista. Todo esto hace sospechar que tienen un mismo origen: la adaptación de los cultos católicos al sistema religioso norteafricano centrado en la estrella Canopo.

Para profundizar en el origen del culto candelariero en Chipude debemos revisar la historia de la parroquia y para ello resulta de gran ayuda el libro de Gloria Díaz y José Miguel Rodríguez, El señorío en las Canarias occidentales ${ }^{24}$. Por lo que sabemos, la iglesia de Chipude es muy antigua: se edificó entre 1530 y 1540, en 1642 obtuvo el título de curato y en 1655 se convirtió en parroquia. ¿Qué ocurre? Pues que el libro menciona que esta no es la primera iglesia de la Candelaria, sino que había otra anterior - llamada Candelaria la Vieja - cerca de la Fortaleza. Si la primera ermita se trasladó a la ubicación actual hacia 1530, Candelaria la Vieja es anterior a 1530. luego es muy, muy antigua.

22 Ver Barrios-Hernández-Trujillo $(2014,2016)$.

23 Perera (2005).

24 Díaz-Rodríguez (1990). 
Cuando leí esto, pensé: no hay dios que la encuentre, vete a saber dónde está, y se lo comenté a Juan Carlos. En una de estas, Juan Carlos fue a jugar un partido de fútbol en la Villa con unos amigos y le preguntó a un jugador de Chipude si sabía dónde estaba la ermita, el chico le respondió que sí, que lo llevaba cuando quisiera. Resulta que todo el mundo en Chipude sabe dónde está Candelaria la Vieja. De hecho, se siguen haciendo procesiones en las que el párroco y los feligreses van desde la iglesia actual hasta los restos de la primitiva ermita.

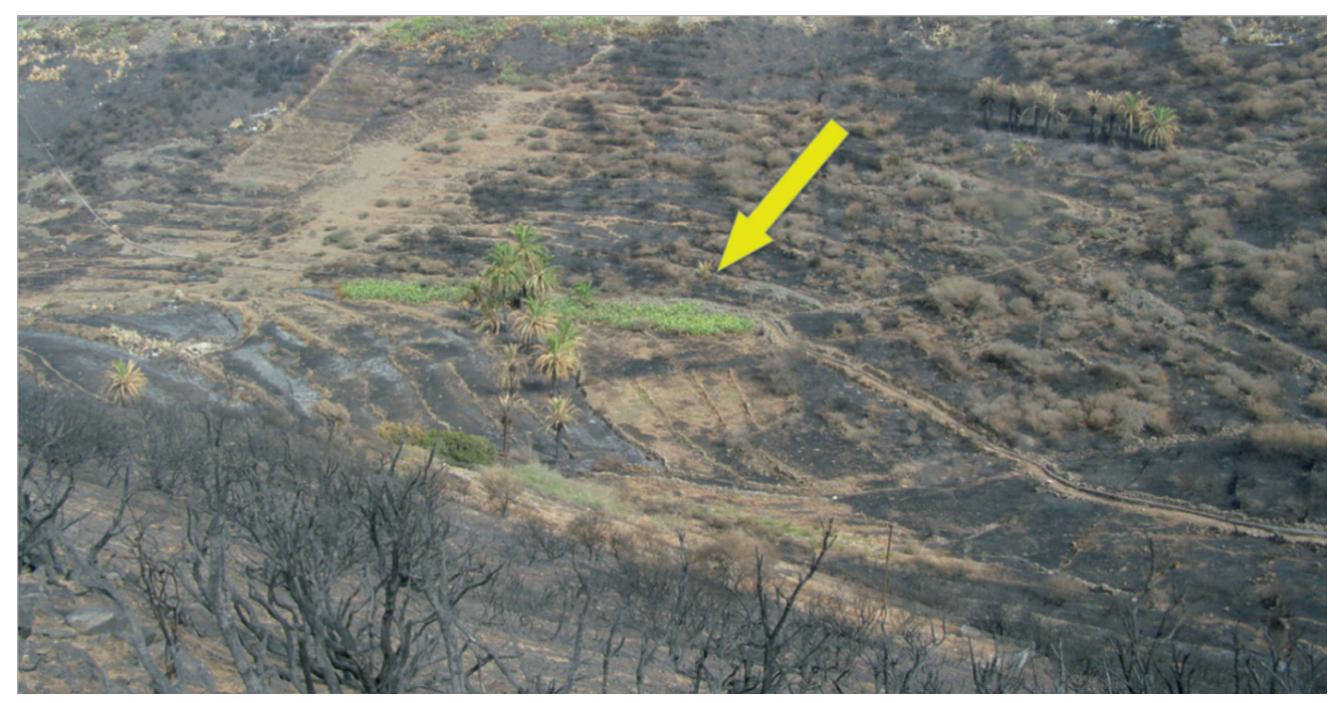

Fig. 9. Localización de Candelaria la Vieja, Chipude (26 de agosto 2012).

En Chipude todo el mundo sabe dónde está Candelaria la Vieja: está en esa vaguada pegada a la Fortaleza, debajo de ese gran manchón de tuneras que ven en la fotografía (Fig. 9), lo único que quedó a salvo del incendio de 2012. Ese es el lugar donde dice la tradición que apareció la Virgen, y, efectivamente, allí encontramos una cruz de madera marcando el sitio, mencionada ya por una descripción del siglo XVIII que se conserva en el Museo Canario. Hay una tradición popular muy fuerte sobre el lugar, por ejemplo, una señora mayor que pastoreaba de niña por esa zona nos dijo que tenían mucho cuidado al pasar por allí -les daba miedo-porque 
decían que había muertos enterrados. Incluso hay una planta que llaman la planta de la Virgen, que dicen que solo nace allí.

Desde el Museo Arqueológico se organizaron tres campañas para limpiar las tuneras en las que se implicó muchísimo la gente de Chipude. En fin, que este es el lugar en el que se dice que apareció la Virgen, seguramente en el siglo XV, en 1400 largos. Una ermita diferente al templo actual, posible fundación franciscana - porque eran los franciscanos los que estaban en la villa de San Sebastián- y situada junto a la Fortaleza.

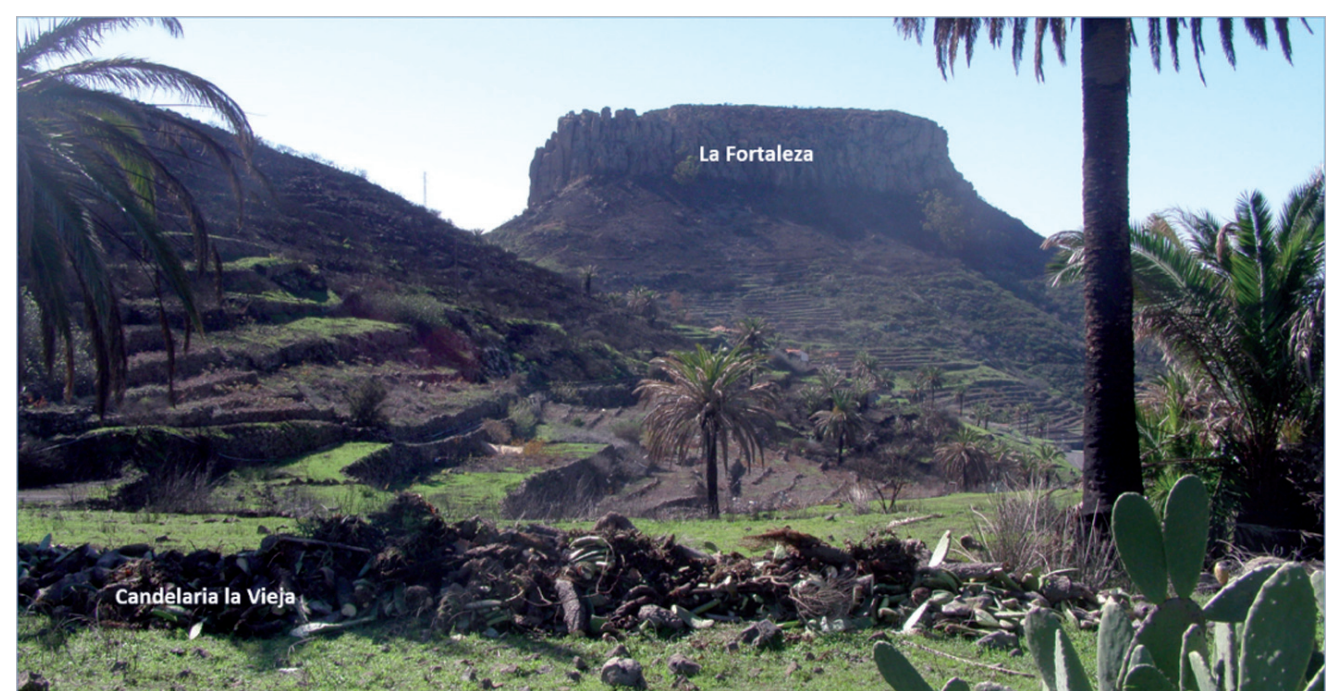

Fig. 10. En primer plano, Candelaria la Vieja. Al fondo, la Fortaleza de Chipude.

Todo esto nos lleva a la Fortaleza de Chipude, epicentro de la religiosidad gomera. Cuando uno piensa en la religión de los gomeros, piensa en la Fortaleza de Chipude, uno de los emblemas, uno de los iconos más importantes del imaginario popular de la isla. Sabemos que fue un centro religioso de los antiguos gomeros porque hay numerosas aras de sacrificio en la explanada superior y porque está relacionada con el solsticio de verano por la tradición popular de subir en la mañana de San Juan para ver salir el sol desde allí. Y ahora resulta que la Fortaleza también está estrechamente 
relacionada con la Candelaria, en primer lugar, por la ermita de Candelaria la Vieja y, en segundo lugar, porque tiene una cueva de San Blas. Y no es una cueva cualquiera.

Se trata de una cueva de grandes dimensiones situada en la ladera occidental que mira a El Hierro y está muy marcada, religiosa y astronómicamente. Por ejemplo, el libro de José Perera que cité antes recoge una entrevista a Dolores Mendoza, de Los Apartaderos - caserío cercano a la Fortaleza - que dice: en la cueva de San Blas los guanches se encontraron a San Blas. Todos los años por el día de San Juan bajábamos; llevábamos un ramito y lo dejábamos allí.. Y Lucio Mendoza Negrín, de Pavón - otro barrio cercano- dice que ... los guanches subían a la cueva de San Blas para adorar al sol, lo contaban los viejos.

Hicimos una investigación de la cueva de San Blas para ver qué podía aportarnos desde el punto de vista astronómico y resultó muy interesante. La cueva es enorme, desde su interior se ve parte de la vertiente occidental de la isla con el barranco de Erque a la izquierda. En el fondo de la cueva hay una especie de hornacina natural donde dicen que estaba san Blas. Subimos en el solsticio de invierno y en la foto (Fig. 11) pueden ver la puesta del sol justo en la mitad de la ladera occidental de la isla de El Hierro: un buen marcador de la parada sur del sol que pudo haber sido utilizado por los gomeros. Más aún, teniendo en cuenta que el otro marcador que hemos encontrado en La Gomera, en las Toscas del Guirre, también es un marcador del solsticio de invierno - se trata de una pequeña cueva que aparentemente no tiene ningún interés, sin embargo, cuando José Miguel y Juan Carlos la prospectaron con detenimiento encontraron un panel con unos 105 caracteres alfabéticos, el mayor panel líbico-bereber que se ha encontrado en Canarias. En la pared enfrentada al panel se abre un hueco ovalado a la altura de los ojos, directamente enfocado al solsticio de invierno. A través del hueco se ve la puesta del sol en el solsticio de invierno 
justo en la iglesia de las Nieves, una de las ermitas más antiguas y más curiosas de la isla ${ }^{25}-$. Es decir, que hay una cierta coherencia en tener dos marcadores del solsticio de invierno.

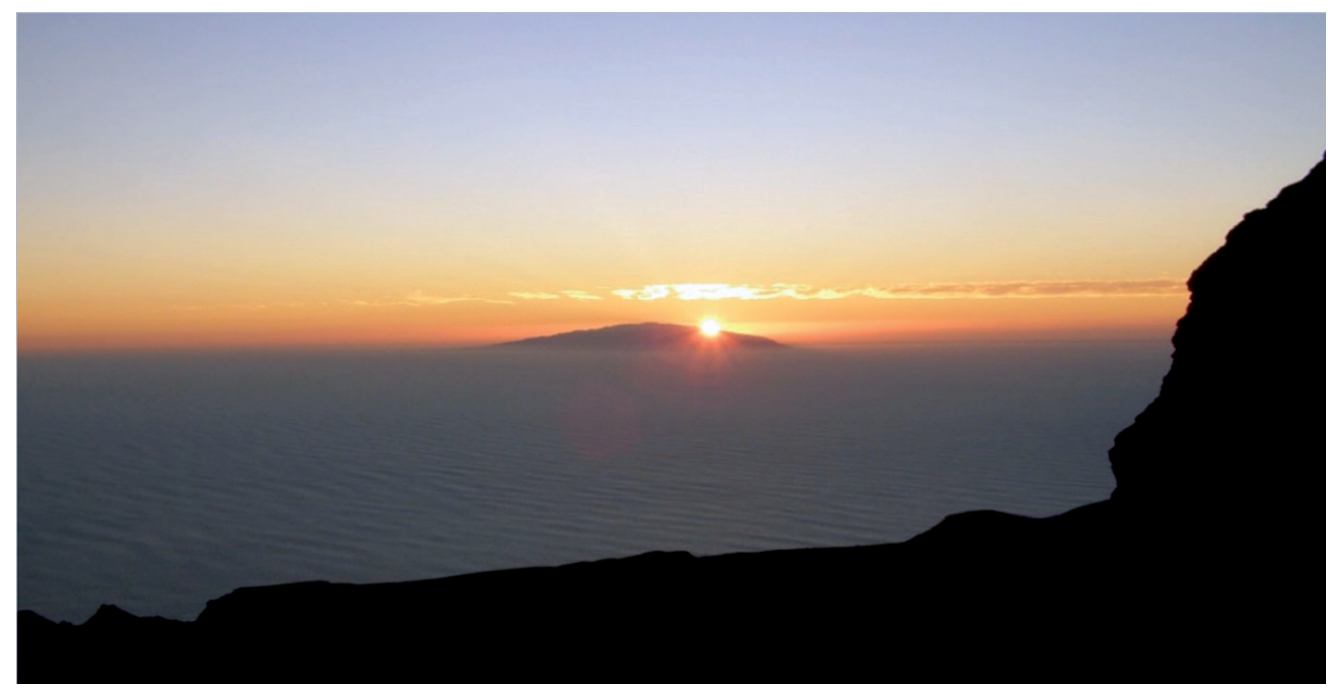

Fig. 11. Puesta del sol en el solsticio de invierno sobre la ladera occidental de El Hierro vista desde la cueva de San Blas (23 de diciembre 2012)

Volviendo a la cueva de San Blas, si analizamos el horizonte astronómico que se ve desde la entrada (Fig. 12), veremos que está completamente enfocada al sur. Sirio, la estrella más brillante del cielo, sale por la montaña de la izquierda y se pone en el mar: no es un buen sitio para observarla porque no ves su orto helíaco lcuando la ves ya está muy alta, por encima de la montaña, y el orto helíaco ha ocurrido varios días antes). Si nos fijamos en la salida y la puesta de sol, tendríamos un buen marcador del solsticio de invierno (SI) sobre la isla de El Hierro, como comenté antes. Sin embargo, para lo que es muy buena la cueva de San Blas es para ver a Canopo. En general, es difícil ver a Canopo porque es una estrella que sale muy al sur y se eleva muy poco, solo se eleva unos $9^{\circ}$ sobre el horizonte. En La Gomera, una isla muy montañosa con

25 Barrios-Marrero-Trujillo (2014). 
muchos barrancos, es difícil encontrar un sitio desde donde puedas verla todo el tiempo. Desde la cueva de San Blas ves todas las fases de Canopo durante todo el año sin ningún problema. Es, por así decirlo, una cueva con vistas a Canopo.

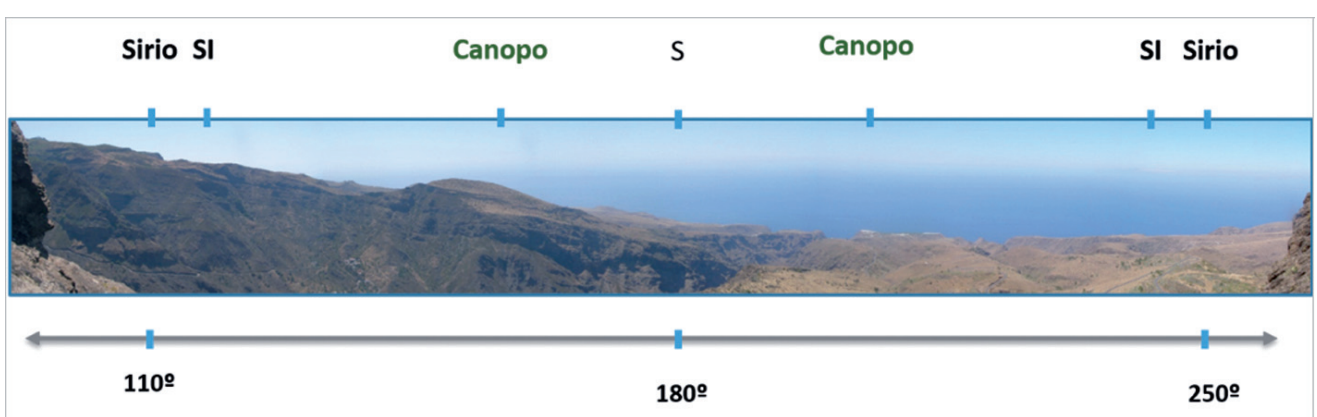

Fig. 12. Horizonte astronómico de la cueva de San Blas (Chipude).

Si algo habla como un pato y camina como un pato, lo más probable es que sea un pato, ¿no es así? En este caso, es mucha casualidad que todo lo que hemos visto vaya en la misma línea, así que uno llega a la conclusión de que esta cueva en la falda de la Fortaleza, marcada astronómicamente por la tradición popular, tiene que ser un lugar de observación de Canopo donde los gomeros estaban llevando el calendario de la isla. La Fortaleza de Chipude ha resultado ser un centro religioso con un fuerte componente astrolátrico.

Visto lo visto - conociendo el trabajo previo de José Perera- nos planteamos hablar con la gente de Chipude para ver si podíamos rescatar algo más de tradición oral sobre estos temas. En junio de 2014, José Miguel Trujillo y Natalia Castel, antropóloga social, llevaron a cabo un trabajo de campo en Chipude entrevistando a gente de la zona. Entre otros vecinos, entrevistaron a Vivita Arteaga (88 años) y su hija Concepción Torres (56 años) y le preguntaron (esto está grabado): 
¿Hubo alguien que se encontró la Virgen [de Candelaria], o eso no se recuerda?

- Los viejos de antes, los pastores que caminaban por ahí. En la Fortaleza hay una cueva que mira para abajo que le dicen la cueva de San Blas y que la gente de antes si subía ahí... y ahí... dicen que se ven unas estrellas, no sé, tres veces al año, que no se ven en otro sitio...

¿Y esa historia, quién se la contaba a usted?

- Las tías de mi padre, las tías de mi padre nos hablaban a nosotras de cosas que ellas tenían...

Vivita, ¿y usted oyó alguna vez lo que acaba de contar su hija de que ahí en la cueva de San Blas miraban una estrella o no sé qué?...

- Sí... iban de madrugada. Yo no me levanté nunca, pero iban de madrugada a verla...

La tradición oral en La Gomera es increíble y que esto se haya mantenido como lo ha hecho es absolutamente sorprendente. Cómo un pueblo ha mantenido durante 500 años la tradición de que debajo de aquellas tuneras estaba la primitiva ermita de la Candelaria. Se dice pronto, 500 años después de la conquista la gente seguía subiendo a la cueva para ver las estrellas... se levantaban de madrugada y subían a la montaña. Fíjense en lo interiorizado que seguían teniendo observar las estrellas desde la cueva.

Pregunta de un asistente: ¿Qué relación tiene San Blas con la Candelaria?

José Barrios: San Blas se celebra el 3 de febrero, el día siguiente de la Candelaria católica...

Un asistente: ... está muy vinculado a ella porque habría curado a un niño con dos candelas y la candela está relacionada con la Candelaria. 
José Barrios: No sabría decirte... podemos reconstruir, meternos en la astrolatría guanche a través de todo esto, pero los mecanismos concretos, los procesos exactos de sincretismo, el papel de fray Alfonso de Bolaños... qué se va haciendo realmente en cada momento... cómo lo van llevando a su terreno... porqué San Blas... en qué momento se introduce cada cosa... Todo eso está por estudiar.

Un asistente: Todo esto es asombroso y demuestra una vez más la importancia de la tradición oral... También me parece importante estudiar cuáles son los componentes, los ingredientes culturales que intervienen para que la gente deje de hacer este tipo de cosas, porque esto hace que se olviden unas cosas y se de origen a otras reinterpretaciones de la Historia, a otras visiones del pasado, casi siempre para justificar su función en el presente.

[Sigue la conferencia]

Bueno, a modo de conclusión parcial: las tradiciones de la Virgen de la Candelaria en Tenerife y La Gomera tienen el mismo origen indígena y demuestran, sin duda, el uso del sistema canopial norteafricano descrito por Viviana Pâques. Por otra parte, este sistema debería proporcionarnos información sobre el calendario, la religión y la organización política, social y territorial de los indígenas. Y, si esto es así, ¿cómo influye en la organización de la isla? ¿Por qué hay cuatro cantones en La Gomera, nueve en Tenerife o doce en Gran Canaria? Si uno lee el trabajo de Pâques, puede encontrar explicaciones muy interesantes.

Por otra parte, todo esto nos da información sobre el proceso de cristianización y sobre el origen y desarrollo de la tradición etnográfica. Nos está explicando qué es lo que está pasando actualmente, cómo se originó y cómo se ha desarrollado. En cualquier caso, todavía queda mucho por estudiar. 


\section{El Pino de Teror}

Para estudiar las tradiciones relacionadas con la Virgen del Pino de Teror, además de las fuentes, los estudios locales y el libro de Viviana Páques, utilizaré las investigaciones de un antropólogo francés, Jean Servier (1918-2000), que trabajó en Argelia a mediados del siglo XX. Esta parte de la conferencia la desarrollé primeramente entre 1987 y $2006^{26}$.

En la pantalla pueden ver el Pino de Teror dibujado desde dos perspectivas distintas: desde el frente y desde la trasera de la iglesia (Fig. 13). Es posible que hayan visto muchas imágenes del Pino, pero estas son las dos únicas auténticas que conocemos, el resto son recreaciones ficticias. Las dibujó Marín de Cubas en 1682, dos años antes de su caída, para ilustrar su manuscrito de 1687. Los dibujos estuvieron perdidos durante mucho tiempo hasta que los encontré en 1990 traspapelados en la Biblioteca Municipal de Santa Cruz de Tenerife. Estaban colocados al final de una de las copias manuscritas de la Historia de Canarias de Pedro Agustín del Castillo. En su momento pensé que podían ser los originales, pero hace unos años se pudo datar la marca de agua del papel hacia $1750^{27}$. Por tanto, son copia de los originales, que siguen perdidos.

Como les decía, toda la iconografía del Pino de Teror que conocemos es completamente imaginaria, salvo estos dos dibujos. ¿En qué consistía este santuario? En primer lugar, tenemos un pino canario enorme. Según cuenta la Información que se instruyó tras su caída en 1684, medía unos 40 metros de altura, algo así como un edificio de 12 pisos, realmente alto. A unos 25-30 metros de altura, donde se ramificaba el pino, estaban plantados tres dragos

26 Ver Barrios (1987, 1993a, 1995, 2006).

27 Eff-Darwich (2014). 
gemelos de una sola copa, tres dragos machos, de unos 2 o 3 metros de altura cada uno ${ }^{28}$. Las fuentes aseguran que los dragos encerraban entre sus raíces una piedra donde se apareció la Virgen y dejó marcadas las huellas de sus pies - es decir, que en la piedra habría un grabado podomorfo.
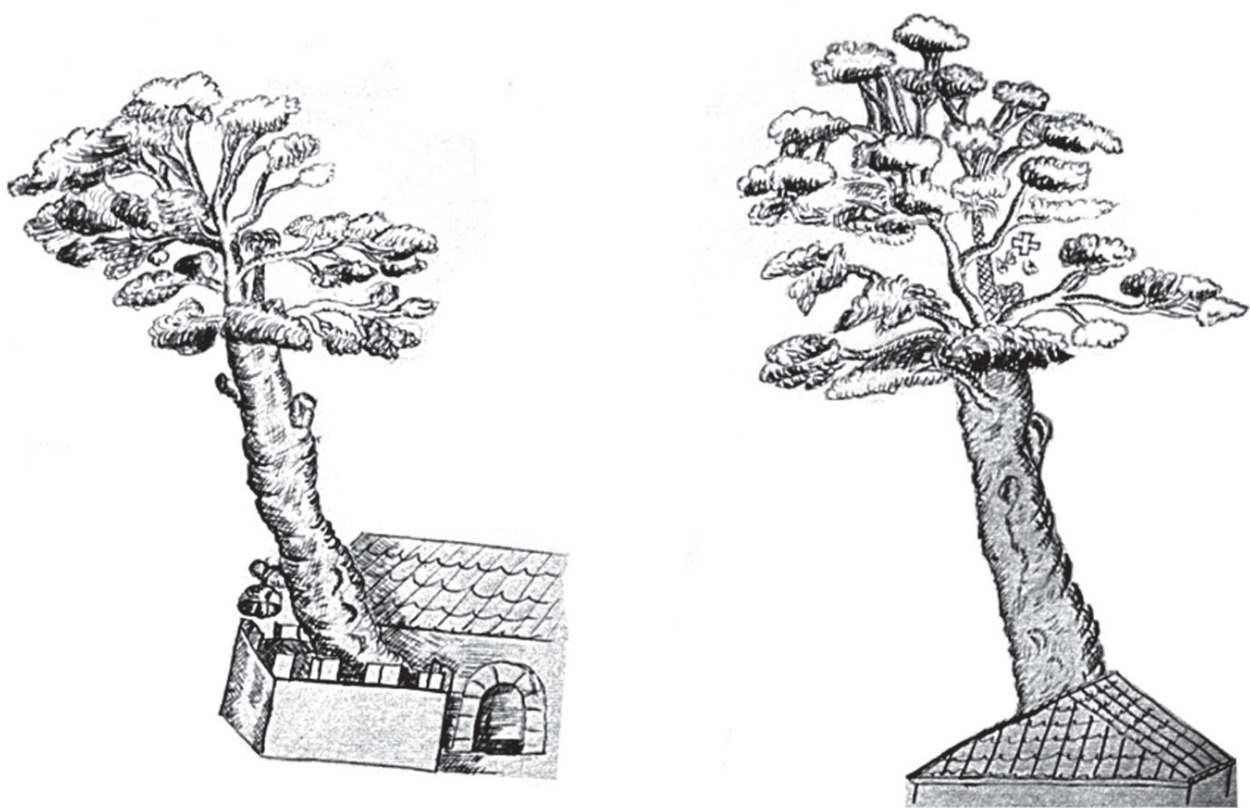

Fig. 13. Dibujos del Pino Teror realizados por Tomás Marín de Cubas en 1682. Copia anónima (Biblioteca Municipal de Santa Cruz de Tenerife)

Al pie del Pino manaba una fuente que los canarios consideraban medicinal y la utilizaban para sanar a sus enfermos. Tras la conquista, un cura le puso la cerca que ven ustedes en el dibujo de la izquierda y empezó a cobrarle a los canarios - a aquellos míseros gentiles- por coger el agua. Cuenta la tradición que por eso se secó la fuente, ahora lo veremos.

28 En 1682 ya solo quedaba un drago. Los otros dos se habían caído durante sendos temporales. 
Claro, uno ve un pino con tres dragos gemelos plantados en la copa a 30 metros de altura, con un grabado podomorfo encerrado entre sus raíces y una fuente manando al pie, donde dicen que se apareció la Virgen antes de la conquista y se pregunta: ¿Qué significa todo esto?... ¿Tiene algo que ver con el triple árbol cósmico descrito por Pâques?... ¿Qué dicen las fuentes?...

Hay varias fuentes antiguas que podemos rescatar. Una es el anónimo de Diego Henríquez. Diego Henríquez (1643-c. 1730) fue un fraile franciscano nacido en Gran Canaria que escribió en 1714 una historia de la religión en las islas y cuando habla de la Virgen del Pino recoge un fragmento anónimo que puede datarse hacia 1640 o poco después. ¿Y qué es lo que dice ese autor anónimo en 1640? Pues dice lo siguiente:

... prosiguiendo Pedro de Vera en el repartimiento... llegó a un término llamado Terori, porque se nombraba así el canario cuyo había sido, del cual tomó el nombre aquel terreno suyo, en el cual apacentaba sus ganados... aviéndoles antes los canarios informado que en aquel sitio de Terori estaba un árbol muy alto y admirable que contenía en sí una rara maravilla, cuya noticia tenían de sus mayores y ancianos y avía más de cien años que venía de unos en otros. ... y que en los ramos de aquel árbol asistía tan continua una estrella muy resplandeciente y clara que ellos la tenían ya por vezina...

Como vemos, el relato anónimo va directamente al grano y sitúa una estrella en el lugar de aparición de la Virgen. En 1687, Marín -que dibujó el árbol y se preocupó por conocer su historia- nos cuenta:

... a el pie de este árbol... salían unos manantiales de agua que... la llebaban los canarios para dar a veber a sus enfer- 
mos... y verdaderamente sanaban. Los spañoles preguntaban la causa, i unos la negaban, i otros decían que allí havía luces de noche, i venido a estar en aquellas tierras de los dragos la madre de Dios, con dos luces encendidas, i otras veses que una estrella estaba muy ordinariamente en aquel citio; no se les daba crédito i llamábanlos de perros idólatras....

En 1737, Pedro Agustín del Castillo (1669-1741) afirma que ... en tiempos de la gentilidad se veía una luz o estrella que alumbraba aquel sitio... Todos estos testimonios relacionan el Pino con luces, con estrellas... con este tipo de cosas. Pero hay una cita muy interesante de Marín de Cubas en 1687 que habla del Pino y lo asocia directamente con la tumba de un varón santo, al señalar:

... lo que piadosamente se tiene es que en aquellas piedras $i$ tierras onde estaban plantados los dragos devía estar el cuerpo de algún varón sancto que en la ysla muriesse.

Y uno se pregunta ¿de dónde sacó esto Marín de Cubas?

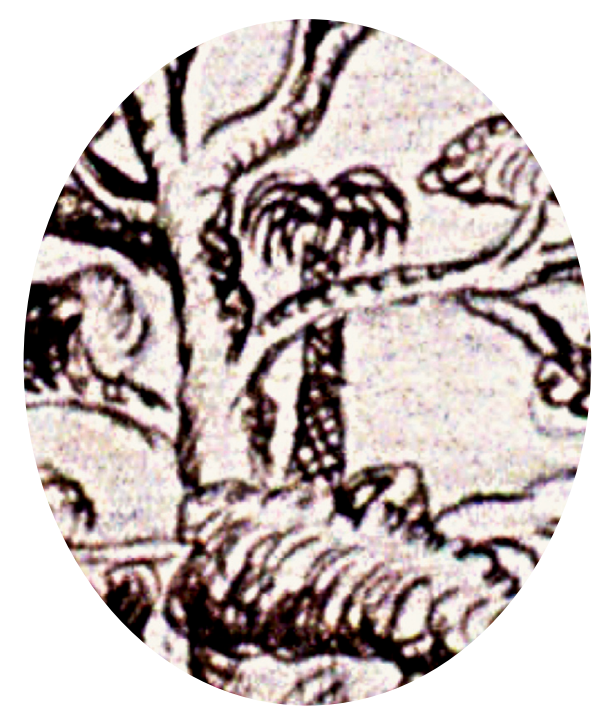

Fig. 14. Drago en la copa del Pino de Teror (detalle). 
La línea historiográfica oficial sobre estas cosas de Marín de Cubas que no se sabe muy bien de dónde salen suele ser muy escéptica - lo digo ya, porque Marín de Cubas ha tenido y sigue teniendo una posición muy peculiar en la historiografía canaria-, sin embargo, veremos en seguida que se trata de una apreciación muy razonable, probablemente tomada de la oralidad de la época. Así que por esta y por otras razones, es uno de mis autores preferidos.

Volviendo a nuestro tema, ¿cómo podemos interpretar este santuario tan particular? Por ejemplo, podemos utilizar los trabajos de campo de Jean Servier sobre el mundo espiritual de los campesinos argelinos, publicados en Les portes de l'année (París, 1962). Un libro extremadamente interesante para los estudios canarios, cuya segunda edición tuve la suerte de comprar en París casi recién salida de la imprenta.

Según este autor, en las concepciones populares del norte de África el cuerpo humano está habitado por dos almas - no una, sino dos-llamadas nefs y rruh. Nefs es el alma vegetativa, le corresponden las pasiones y los comportamientos emocionales, es femenina, húmeda y fija. Rruh es el alma sutil o aliento, le corresponde la voluntad, es masculina, seca y móvil. En el plano de los símbolos materiales, la unión de estas dos almas, principios esenciales de la persona humana, está representada por la pareja árbol-roca. Un árbol encerrando entre sus raíces una roca representa la unión sagrada de los dos principios, nefs y rruh. Su acoplamiento constituye por sí solo lo esencial de un santuario...29.

Si retrocedemos y recordamos lo que decía Marín en 1687: ... lo que piadosamente se tiene es que en aquellas piedras i tierras onde

29 Servier (1985: 10-24). Resumo muchísimo su trabajo, cuya lectura recomiendo encarecidamente. 
estaban plantados los dragos devía estar el cuerpo de algún varón sancto... Y lo analizamos a la luz del testimonio argelino de Servier, observaremos que en el Pino no estaba la tumba material, sino la tumba simbólica de un varón santo ${ }^{30}$.

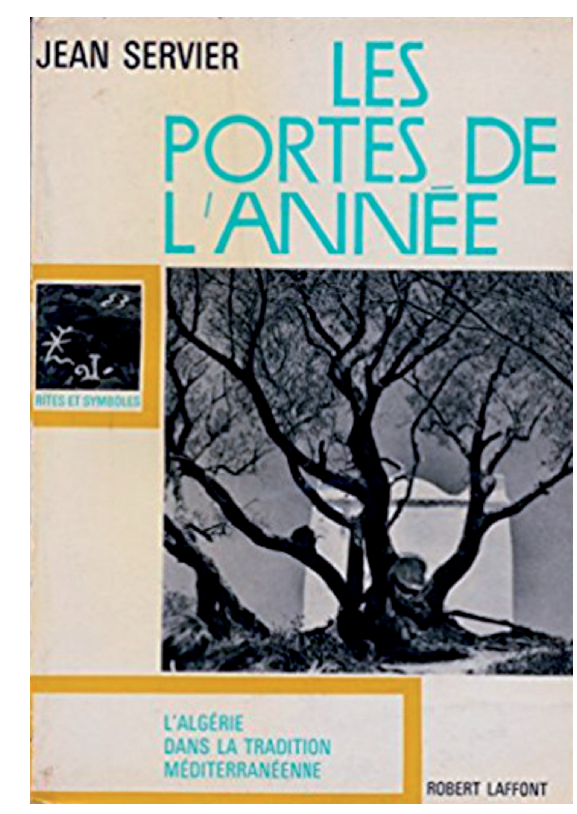

Fig. 15. Portada de la primera edición (Paris, 1962)

De hecho, la portada de la primera edición del libro de Servier (Fig. 15) muestra un árbol encerrando una piedra entre sus raíces, la tumba simbólica del santo protector de una villa argelina de las muchas que documenta en su trabajo. Cuando el árbol se seca o se deteriora y corre peligro de caer, lo sujetan para que no se caiga, porque su caída supone la pérdida de la protección del santo. En este sentido, si leen la carta del cura de Teror al provisor de la diócesis tras la caída del Pino, da la impresión de que se les había acabado el mundo, el pueblo estaba destrozado por el suceso. En esencia, esto fue lo que presenté en el III Congreso Iberoamericano de Antropología en 1987, allí sostuve que en el Pino de Teror estaba 
simbolizada la tumba de un hombre santo, llamado - no tenemos otro nombre- Aterure.

\section{Auge tardío del culto}

Sobre el culto católico en Teror hay una cosa muy interesante: su importancia se fraguó mucho después de la conquista de la isla y en circunstancias muy poco claras. Mientras que la notoriedad del culto católico a la Candelaria en Tenerife y en La Gomera es coetánea o anterior a la propia conquista, el culto a la Virgen del Pino de Teror en Gran Canaria solo empieza a tomar relevancia a comienzos del siglo XVII.

Fíjense ustedes: el propio Bartolomé Cairasco de Figueroa (15831610), cuando escribió su famoso Templo militante -cuatro tomos dedicados a glosar las festividades del calendario religioso a lo largo de todo el año-, el 2 de febrero habla de la Candelaria de Tenerife y del milagro de su aparición, pero el 8 de septiembre no dice nada sobre la Virgen del Pino. Cairasco, uno de los máximos representantes del catolicismo institucional en la isla, no menciona en su obra a la Virgen del Pino. ¿Cómo se explica?

Veamos rápidamente la cronología del culto. El primer documento conocido que nombra la ermita de Santa María de Therore es de 1514 y recoge su anexión a la fábrica de la catedral de Las Palmas $^{31}$. La catedral tardó mucho tiempo en edificarse, costó mucho dinero y mucho esfuerzo. Se inauguró, por fin, la víspera de Corpus del año 1570. En 1595 la ermita estaba en muy mal estado, a punto de caerse. En 1607 tiene lugar la primera bajada de la Virgen del

31 El clero de Gran Canaria estaba muy interesado en sacar adelante la catedral, así que anexionaron diversas ermitas de la isla para detraer sus rendimientos y utilizarlos en la construcción. 
Pino a Las Palmas. En 1609, Cairasco no la nombra en la tercera parte de su Templo militante. En 1615 se producen las primeras noticias sobre las romerías del 8 de septiembre. A partir de estas fechas su importancia crece de forma imparable hasta alcanzar su esplendor en el siglo XVIII con la fábrica de la tercera iglesia en 1750 y la declaración como patrona de Gran Canaria en 1766.

Sin duda, hace falta explicar por qué Teror - un sitio húmedo, con malos caminos, alejado de la ciudad, con una ermita medio en ruinas a la que no se le hace el más mínimo caso- se convierte en pocos años en el principal centro católico de la isla a costa de la recién inaugurada catedral de Las Palmas. Hay que explicar por qué la Iglesia se ve obligada a abandonar el culto principal de la isla en la catedral - que le había costado tanto construir-y trasladar el centro religioso a Teror. Los motivos tuvieron que ser muy importantes y de mucho peso ${ }^{32}$.

Mi explicación es la siguiente ${ }^{33}$ : en Teror existía un culto canopial de los antiguos canarios, lo vemos por las fechas, el pino, la estrella, la tumba del santo... Todo indica que allí existía un centro de culto relacionado con Canopo: el sistema astrolátrico que ya conocemos $^{34}$. Tras la conquista, ese culto decayó por los efectos propios de la misma, pero se reactivó a finales del siglo XVI, prin-

32 Para otras explicaciones alternativas y/o complementarias a la que propongo, ver el interesante trabajo de G. A. Trujillo (2010) y la bibliografía a la que remite. Hasta donde yo sé, ninguna de estas alternativas explica por qué se eligió la fecha del 8 de septiembre para celebrar la fiesta de la Virgen del Pino.

33 Naturalmente, se trata de una explicación que habrá que modular, matizar y corregir en función del desarrollo de la investigación. No obstante, no encuentro otra explicación a lo sucedido que sea coherente con todos los hechos.

34 En realidad, hay motivos para sospechar que en Gran Canaria pudieron coexistir dos sistemas astrolátricos distintos. Uno de ellos sería el culto a Canopo que venimos tratando, el otro un posible culto a Sirio relacionado con la cultura de los guanartemes y los faicanes. Ver al respecto, Barrios (2004: 128). 
cipios del XVII. Una vez pasado el trauma inicial de la conquista, la isla se fue aclimatando al nuevo orden social y, bueno, retomó antiguas costumbres como podía ser ir a Teror por el orto helíaco de Canopo. Las importantes decisiones que tuvo que tomar la Iglesia para controlar lo que estaba sucediendo subrayan la importancia que tomó está costumbre.

Antes de 1582 el orto helíaco de Canopo tenía lugar a mediados de agosto, pero después de 1582 la reforma gregoriana del calendario retrasó la fecha 10 días, por tanto, a comienzo de 1600 las concentraciones populares debían de estar ocurriendo a finales de agosto/principios de septiembre. La Iglesia se vio obligada a tomar decisiones importantes porque la religiosidad popular de la isla se le estaba escapando de las manos, tenían que hacer algo y pensaron: ¿cómo arreglamos este problema? La solución estaba clara: promover el culto católico del lugar. Podían haber llevado la celebración al 15 de agosto, como hicieron en Tenerife y La Gomera, pero decidieron llevarla al 8 de septiembre, Natividad de la Virgen, otra fiesta importante de la Iglesia muy cercana a la celebración popular. Por esta razón instituyeron las fiestas del 8 de septiembre, cuyas primeras noticias aparecen hacia 1615.

Lo que sabemos es que este proceso de sincretismo se produjo mucho más tarde que en Tenerife y La Gomera. Fue la necesidad de integrar en el sistema católico la reactivación del culto indígena en aquella zona lo que llevó a la Iglesia a olvidarse del culto oficial en la catedral y controlar lo que estaba pasando en Teror.

En resumen, el Pino era un santuario de los antiguos canarios donde se celebraban fiestas canopiales a mediados de agosto. Tras la conquista el culto se mantiene latente. Hacia 1600, el culto se reactiva y los canarios vuelven a Teror por la reaparición de Canopo, ahora a finales de agosto. La Iglesia necesita controlar la 
situación e implanta la celebración de la Natividad de la Virgen, el 8 de septiembre, lo que no deja de tener bastante sentido: nace la estrella y nace la Virgen.

Al final, resulta ser una fiesta muy parecida formalmente a las fiestas de la Candelaria en Tenerife y La Gomera: es la fiesta más importante de la isla, se celebra el 8 de septiembre - cercana al orto de Canopo-, hay una concentración de la población de la isla, es de origen indígena anterior al siglo XV y está relacionada con el Pino de Teror - un santuario canario asociado con una estrella y la tumba de un santo-. Todo esto señala el culto a Canopo en Teror.

En conclusión: Tenerife, La Gomera y Gran Canaria, tres cristianizaciones distintas del mismo culto indígena a la estrella Canopo. Muchas gracias por su atención. 


\section{REFERENCIAS}

Barrios García, José (1987) “La Virgen del Pino de Teror (islas Canarias): adaptación católica de un culto africano". Comunicación al IV Congreso Iberoamericano de Antropología (Las Palmas, 1987). Sin publicar.

Barrios García, J. (1993a) “Dos dibujos del pino de Aterure”. En G. Díaz y F. González (eds.), Strenae Emmanuelae Marrero oblatae. La Laguna: Universidad, vol. 1, pp. 111-130. https://academia. edu/04176385.

Barrios García, J. (1993b) “A pre-16th century Berber solstitial marker on Grand Canary island (North West Africa)". En W. B. Murray \& A. Stoev (eds.), Proceedings of the 4th Oxford International Conference on Archaeoastronomy (Stara Zagora, 1993). Sin publicar. https://academia. edu/04176408.

Barrios García, J. (1995) “Estudio sobre la noción de alma entre los antiguos canarios". En Proceedings of the Ist World Congress on Mummy Studies (Puerto de la Cruz, 1992). Santa Cruz de Tenerife: Museo Arqueológico y Etnográfico, vol. 2, pp. 683-690. https://academia.edu/04176396.

Barrios García, J. (1996) "The Guanche lunar calendar and the Virgin of Candelaria (Tenerife, 14th-15th centuries)". En W. Schlosser (ed.), Proceedings of the Second SEAC Conference (Bo- 
chum, 1994). Bochum: Astronomisches Institut der Ruhr-Universität, pp. 151-162. https://academia.edu/00775626.

Barrios García, J. (2004 [1997]) Sistemas de numeración y calendarios de las poblaciones bereberes de Gran Canaria y Tenerife en los siglos XIV-XV. La Laguna: Servicio de Publicaciones de la Universidad de La Laguna. [Tesis doctoral]. https:// academia.edu/00776580.

Barrios García, J. (2006) “El pino de Aterure”. El Pino. Programa-revista de las Fiestas del Pino (Teror, Gran Canarial, vol. 55, pp. 16-17. https://academia.edu/03093822.

Barrios García, J. (2015) "Mathematics and astronomies of the ancient Berbers". En H. Selin (ed.), Encyclopaedia of the history of science, technology, and medicine in non-Western cultures (3rd edition). Berlin: Springer, pp. 1-16. https://academia.edu/16917387.

Barrios García, J.; Hernández Marrero, J. C.; Trujillo Mora, J. M. (2014) "Investigaciones arqueoastronómicas en La Gomera. El solsticio de invierno en las Toscas del Guirre". En XX Coloquio de Historia Canario-Americana (Las Palmas, 2012). Las Palmas de Gran Canaria: Cabildo de Gran Canaria, pp. 1327-1334. https://academia. edu/04072735.

Barrios García, J.; Hernández Marrero, J. C.; Trujillo Mora, J. M. (2016) "Investigaciones arqueoas- 
tronómicas en La Gomera. La cueva de San Blas y el origen del culto a la Candelaria en Chipude". En XXI Coloquio de Historia Canario-Americana (Las Palmas, 2014). Las Palmas de Gran Canaria: Cabildo de Gran Canaria, vol. 21(80), pp. 1-12. https://academia.edu/08963270.

Basset, R. (1910) "Recherches sur la religion des berbères". Revue de l'Histoire des Religions (Paris), vol. 61, pp. 291-342. https://gallica.bnf.fr/ ark:/12148/bpt6k104967s/f004.

Camps, G. (1980) Berbères, aux marges de l'histoire. Toulouse: Ed. des Hespérides.

Camps, G. (1988) “Animisme”. En Encyclopédie Berbère, Anacutas - Anti-Atlas. Aix-en-Provence: Edisud, vol. 5, pp. 660-672. https://encyclopedieberbere.revues.org/2509.

Charles-Picard, G. (1954). Les religions de l'Afrique antique. Paris: Libraire Plon.

Cubillo Ferreira, A. L. (1985) Antropónimos guanches y berberes. Santa Cruz de Tenerife - Las Palmas de Gran Canaria: Centro de Estudios Africanos.

Díaz Padilla, G.; Rodríguez Yanes, J. M. (1990). El señorío en las Canarias occidentales. La Gomera y El Hierro hasta 1700. El Hierro-La Gomera: Cabildo de El Hierro-Cabildo de La Gomera. https:// academia.edu/38171306. 
Eff-Darwich Peña, Á. I. (2014) “Estudio codicológico de tres manuscritos de la Biblioteca Municipal de Santa Cruz de Tenerife". En XX Coloquio de Historia Canario-Americana (Las Palmas, 2012). Las Palmas de Gran Canaria: Cabildo de Gran Canaria, pp. 473-484. http://coloquioscanariasamerica.casadecolon.com/index.php/CHCA/ article/view/9188

García Pulido, D.; Bolaños González, J. I.; Paz Sánchez, M. de (eds.). (2019). Amaro Pargo. Documentos de una vida. Volumen 5. San Amaro bendito. Santa Cruz de Tenerife - Las Palmas de Gran Canaria: Ediciones Idea. https://www.academia. edu/41340465.

Hawkins, G. S.; Roshental, S. K. (1967) “5,000- and 10,000-year star catalogs". Smithsonian Contributions to Astrophysics (Washington), vol. 10, núm. 2. pp. [i-iv] + 141-179. https://doi.org/10.5479/ si.00810231.10-2.141.

Pâques, V. (1964) L'arbre cosmique dans la pensée populaire et dans la vie quotidienne du nord-ouest africain. Paris: Institut d'Ethnologie (Musée de l'Homme).

Perera López, J. (2005) La toponimia de La Gomera. Un estudio sobre los nombres de lugar, las voces indígenas y los nombres de plantas, animales y hongos de La Gomera. La Gomera: Asociación Insular de Desarrollo Rural. 4 tomos en 25 vol. (DVD). 
Santana Rodríguez, L. (2018) "Cuando las romeras cruzaban la cumbre para cumplir con la Candelaria". En C. Rodríguez (ed.), Imagen y reliquia. Nuevos estudios sobre la antigua escultura de la Candelaria. La Laguna: Ayuntamiento, pp. 46-59. https://academia.edu/38050223.

Schaefer, B. E. (1985) "Predicting heliacal risings and settings". Sky \& Telescope (Cambridge MA), vol. 70, pp. 261-263.

Schaefer, B. E. (1987) "Heliacal rise phenomena". Archaeoastronomy (Cambridge), vol. 11, pp. S19-S33.http://articles.adsabs.harvard.edu/ pdf/1987JHAS...18...19S.

Servier, J. (1985) Tradition et civilisation berbères. Les portes de l'année (2e éd.). Mónaco: Éditions du Rocher.

Trujillo Yánez, G. A. (2010) “La advocación de Nuestra Señora del Pino de Teror (Gran Canaria) ¿Una divinidad sincrética?". En XVIII Coloquio de Historia Canario-Americana (Las Palmas, 2008). Las Palmas de Gran Canaria: Cabildo de Gran Canaria, pp. 255-271. http://coloquioscanariasamerica.casadecolon.com/index.php/CHCA/article/view/8952 